\title{
High Calcium Score Predicts Severity of the Culprit Lesions in Patients with Acute Coronary Syndromes
}

\author{
Jakó Beáta1, Benedek Theodora², Suciu Zsuzsanna², Benedek $1^{2}$ \\ Clinic of Cardiology, County Emergency Clinical Hospital, Tîrgu Mures, Romania \\ 2 Clinic of Cardiology, Discipline of Internal Medicine 6, University of Medicine and Pharmacy, Tîrgu Mureș, Romania
}

Introduction: Coronary calcium score, as determined by Angio CT multislice, has been proved to represent a reliable parameter which reflects the global cardiovascular risk. We aimed to study the characteristics of culprit lesions in Acute Coronary Syndrome (ACS) patients with low versus high calcium score.

Material and methods: A total of 45 patients with ACS underwent 64-slice CCTA. Group 1 - 19 patients with Ca score below $400 H U$, Group $2-26$ patients with calcium score $>400 \mathrm{HU}$. In all patients a complex CT analysis of the culprit plaque was performed.

Results: There were no significant differences between the groups at baseline as regard to age, gender, cardiovascular risk factors ( $p>0.2)$. In patients with high calcium score, culprit lesions presented a significantly larger amount of plaque burden than in patients with low calcium score $(82.8 \mathrm{ml}$ versus $131.81 \mathrm{ml}, \mathrm{p}<0.0001)$. This was also true when assessing in a subanalysis different cut-off points for definition of relatively higher calcium score $(89.66 \mathrm{ml}$ versus $137.93 \mathrm{ml}, \mathrm{p}<0.0001$, for calcium score cut off $600 \mathrm{HU}, 97.88 \mathrm{ml}$ versus $137.57 \mathrm{ml}, \mathrm{p}<0.0001$ for calcium score cut-off of $1000 \mathrm{HU})$.

Conclusion: Our data shows that patients with high calcium score who develop an acute coronary syndrome present larger atheromatous plaque than those with low calcium scores, and theseverity of the culprit lesions correlates with global cardiovascular risk as expressed by a high calcium score.

Keywords: plaque burden, calcium scoring, cardiovascular risk score

Received: 11 June 2013

\section{Introduction}

Coronary artery disease continues to represent the major cause of death worldwide despite major measures addressed to cardiovascular risk reduction. The most devastating manifestation of a coronary artery disease is represented by an acute coronary syndrome, which is usually triggered by the rupture of a vulnerable, unstable plaque.

Detection of unstable plaques is of extreme importance for initiation of adequate therapeutic measures addressed to plaque stabilization in order to prevent plaque rupture with the consequent the development of an acute coronary syndrome. There are several techniques currently used for in-vivo detection of unstable plaques (most used being intravascular ultrasonography with virtual histology - VHIVUS, Optical Coherence Tomography - OCT and Multislice Cardiac Computed Tomography), based on several markers of instability associated with vulnerable plaques, such as:

- detection of a thin cap fibroatheroma (usually detected by Optical Coherence Tomography);

- positive remodeling of the coronary artery at the site of the vulnerable plaque (identifiable with IVUS, OCT or Cardiac Computed Tomography);

- spotty calcifications within the plaque (identifiable mainly by Cardiac Computed Tomography);
- presence of a low density lipid core by Cardiac CT or a necrotic core by VH-IVUS.

Among these markers of plaque vulnerability a critical role is played by the low density lipid core within the plaque, or the burden with low density atheroma, which has been demonstrated to represent the most unstable part of the plaque. It has been shown that unstable plaques are associated with higher burden with low density atheroma, as identified by Cardiac CT.

However, it is well known that not all the vulnerable plaques trigger an acute coronary syndrome. Serial IVUSbased studies suggested thatanunstable lesion may progress into a stable one in a very short period and vice versa, a stable plaque into an unstable one. Therefore, a comprehensive risk assessment should also take into consideration other risk factors, beside the culprit plaque characteristics. One of these risk factors, proposed in this study, is represented by the coronary calcium score, score which is calculated by Multislice Cardiac CT, expressing the global burden with calcium of the coronary tree. It has been clearly demonstrated that a high calcium score is associated with an increased cardiovascular risk, a value of more than 400 for Calcium score being considered as a marker of increased cardiovascular risk.

However, no study so far has evaluated the correlation between the calcium score, as a marker of global cardiovascular risk, and coronary plaque characteristics, mainly the severity of the culprit lesions in acute coronary syndromes. 
The aim of this study was to show that a high calcium score is associated with a more severe evolution of the coronary plaques especially in acute coronary syndromes, as expressed by a larger plaque volume and a higher burden with low density atheroma (marker of plaque vulnerability) at the site of the culprit lesion.

\section{Material and methods}

\section{Patient population}

The study population consists in 45 patients with acute coronary syndrome (22 with unstable angina pectorisand 23 with non-ST-segment elevation myocardial infarction). Patients were enrolled in the study if they had a chest pain and ST depression associated with elevation of troponin value or if they presented a new ischaemic discomfort that was Canadian Cardiovascular Society class 3 or 4 associated with ECG changes suggestive of ischaemia.

In all patients we performed urgent Multislice Cardiac Computed Tomography, including calculation of coronary calcium scoring and complex plaque analysis, followed by invasive coronarography, to identify, characterize and treat the culprit lesions.

According to their calcium score, the patients were distributedinto two groups: group $1-19$ patients having a coronary Ca score below 400, and group $2-26$ patients with Coronary Ca score higher than 400 .

Patients with: cardiac arrhythmias, renal insufficiency (glomerular filtration rate $<30 \mathrm{ml}$ ), allergy to iodine contrast media, or inadequate image quality because of motion artifacts were excluded from the study. Written informed consent was obtained from all study patients.

\section{Methods}

Cardiac CT multislice analysis was performed in all patients using a multislice 64 cardio CT equipment (Somatom Sensation, Siemens).Patients were pre-scanned to determine the total calcium scoring (Agatson score) of the coronary tree. Before examination the patient's heart rate were reduced to the threshold of 65 b.p.m. with administration of beta-blockers (Metoprolol 50-100 mg, oral). The angiographic CT scan was made with a dual phase administration of contrast media (iopromide $370 \mathrm{mg} / \mathrm{ml}$ ) in an antecubital vein, at a rate of $4.8 \mathrm{ml} / \mathrm{sec}$. A standard MDCT acquisition protocol were used to determine coronary artery calcium score and to made different multiplanar reconstruction to coronary arteries. The coronary artery tree model was based on the 17-segment coronary artery scheme proposed by the American Heart Association. The complex plaque analysis included determination of plaque volumes $(\mathrm{ml})$, followed by calculation of burden with different plaque components according to plaque density expressed in Hounsfield Units - HU (burden with low density plaque below $30 \mathrm{HU}, 60 \mathrm{HU}$ and $100 \mathrm{HU}$ ).

In all causes the quantitative coronary angiography was performed on the sameor second day after MDCT exami- nation. Segmental coronary artery stenosis was analyzed in different projections using the same coronary artery tree models, and the appropriate PCI and stenting was performed.

\section{Statistical analysis}

Statistical analysis was performed using Graph InStat Pad softwares. Continuous values are expressed as the mean and standard deviation, and statistical significance was determined using the Mann-Whitney test. Categorical variables are expressed as percentages and were compared using Fisher's exact test. Statistical significance was considered for a $\mathrm{p}$ value $<0.05$, and all $\mathrm{p}$ values were 2 -sided.

\section{Results}

\section{Baseline characteristics of study population}

There were no significant differences in baseline characteristics of study population between the two groups, as regards to age, gender or conventional cardiovascular risk factors (Table I).

The mean plaque volume of the culprit lesion showed a significant difference between the 2 groups $-82.8 \mathrm{ml}$ $\pm 38.33 \mathrm{ml}, 95 \%$ CI $64.32-101.28 \mathrm{ml}$ in group 1 and $131.80 \mathrm{ml} \pm 39.88 \mathrm{ml}, 95 \% \mathrm{CI} 115.69-147.92 \mathrm{ml}$ in group 2 , $(\mathrm{p}=0.0002)$ (Table II).

We performed a sub-analysis taking into consideration different cut-off points for defining a high calcium score: 600, respectively 1000 and we recoded similar results: plaque volume of the culprit lesion was significantly higher in cases with high calcium score $(89.66 \mathrm{ml}$ versus 137.93 $\mathrm{ml}, \pm 40.05 \mathrm{ml}$ versus $38.52 \mathrm{ml}, 95 \%$ CI $73.12-106.20$ versus $119.90-155.96, \mathrm{p}<0.0004$, for a 600 cut-off of calcium score, $97.88 \mathrm{ml}$ versus $137.57 \mathrm{ml}, \pm 45.14 \mathrm{ml}$ versus $35.6 \mathrm{ml}, 95 \% \mathrm{CI}$ 81.03-114.74 versus $117.85-157.29$, $\mathrm{p}$ $<0.0040$ for calcium score cut-off of 1000)(Figure 1).

Quantitative plaque analysis including calculation of burden with low density plaque was performed according to different cut-off points for defining the plaque as "low density" - $30 \mathrm{HU}, 60 \mathrm{HU}$ and $100 \mathrm{HU}$, indicating that a higher burden with below $30 \mathrm{HU}$ plaque is significantly associated with higher calcium score in all cases, no matter

Table I. Baseline characteristics of study population

\begin{tabular}{lccc}
\hline $\begin{array}{l}\text { Baseline } \\
\text { characteristic }\end{array}$ & $\begin{array}{c}\text { Gr. } 1-\text { Ca score }<400 \\
\mathrm{n}=19\end{array}$ & $\begin{array}{c}\text { Gr. 2-Ca score }>400 \\
\mathrm{n}=26\end{array}$ & P value \\
\hline $\begin{array}{l}\text { Age, years, } \\
\text { mean } \pm \text { SD }\end{array}$ & $62.42 \pm 9.2$ & $65.19( \pm 11.8)$ & 0.25 \\
Gender, male & $16(84.2 \%)$ & $19(73.0 \%)$ & 0.54 \\
Diabetes & $3(15.7 \%)$ & $4(15.3 \%)$ & 0.1 \\
Hypertension & $16(84.2 \%)$ & $18(69.2 \%)$ & 0.31 \\
Obesity & $7(36.8 \%)$ & $13(50.0 \%)$ & 0.185 \\
Smoker & $14(73.6 \%)$ & $16(61.5 \%)$ & 0.72 \\
Dislipidemia & $10(52.6 \%)$ & $10(38.4 \%)$ & 0.6 \\
Arteriopathy & $1(5.2 \%)$ & $1(3.8 \%)$ & 0.9 \\
Stroke & 0 & $3(11.5 \%)$ & 0.22 \\
TOTAL & $19(42.2 \%)$ & $26(57.5 \%)$ & 0.09 \\
\hline
\end{tabular}


Table II. The mean plaque volume of the culprit lesion (SD - Standard deviation, $95 \% \mathrm{Cl}-95 \%$ confidence interval)

\begin{tabular}{lcccccc}
\hline & $<400$ & $>400$ & $<600$ & $>600$ & $<1000$ & $>1000$ \\
\hline Plaque volume $(\mathrm{ml})$ & 82.8 & 131.8 & 89.6 & 137.93 & 97.88 & 137.57 \\
SD & 38.33 & 39.88 & 40.05 & 38.52 & 45.14 & 35.6 \\
95\% Cl & $64.32-101.28$ & $115.69-147.92$ & $73.12-106.20$ & $119.90-155.96$ & $81.03-114.74$ & $117.85-157.29$ \\
P value & \multicolumn{2}{c}{0.0002} & & & 0.0004 & \\
\hline
\end{tabular}

if we consider the cut off for "high calcium score" at values of 400,600 or 1000 .

For patients with calcium score below 400, the mean burden with atheroma below $30 \mathrm{HU}$ was $15.2 \mathrm{ml}$ compared with $29.27 \mathrm{ml}$ in those with $\mathrm{Ca}$ score $>400$ with 95\% CI 7.8-20.34, p value highly significant: $<0.0001$. Taking into account a cut-off point of $60 \mathrm{HU}$ for "low density" atheroma, the difference was still statistically significant: $26.97 \mathrm{ml}$ in group 11 versus $38.21 \mathrm{ml}$ in group 2 , 95\%CI 4.65-17.81, $\mathrm{p}=0.0013$. The same was true in case of $<100 \mathrm{HU}$ plaque burden: $41.31 \mathrm{ml}$ versus $53.07 \mathrm{ml}$, 95\%CI 2.18-21.33, $\mathrm{p}=0.0173$ (Figure 2A).

We observed the same differences for a calcium score cut-off value of 600: highly statistical significant differences between the groups for $<30 \mathrm{HU}$ the culprit lesion plaque volume were $18.09 \mathrm{ml}$ versus $29.88 \mathrm{ml}(\mathrm{p}=0.0002)$, for $<60 \mathrm{HU} 29.85 \mathrm{ml}$ versus $37.97 \mathrm{ml}(\mathrm{p}=0.023)$, and for $<100 \mathrm{HU} 43.63 \mathrm{ml}$ versus $52.71 \mathrm{ml}(\mathrm{p}=0.03)$ (Figure 2B).

With a calcium score cut-off point of 1000 , the difference between the groups remained significant only for plaque density $<30 \mathrm{HU}-20.5 \mathrm{ml}$ versus $28.99 \mathrm{ml}(\mathrm{p}=$ 0.0047), while for the rest of the cases the differences between the groups was not anymore significant: $31.93 \mathrm{ml}$ versus $36.53 \mathrm{ml}(\mathrm{p}=0.23)$ for $<60 \mathrm{HU}, 46.25 \mathrm{ml}$ versus $51.82 \mathrm{ml}(\mathrm{p}=0.14)$ for $<100 \mathrm{HU}$ (Figure 2C).

\section{Discussion}

This study shows that in patients with high coronary calcium score level, the identified culprit lesion presented large volumes and high low-density atheroma burden. Several studies published in the literature in the last years indicated that vulnerable plaque are associated with larger plaque volumes and the larger is this volume, the more vulnerable is

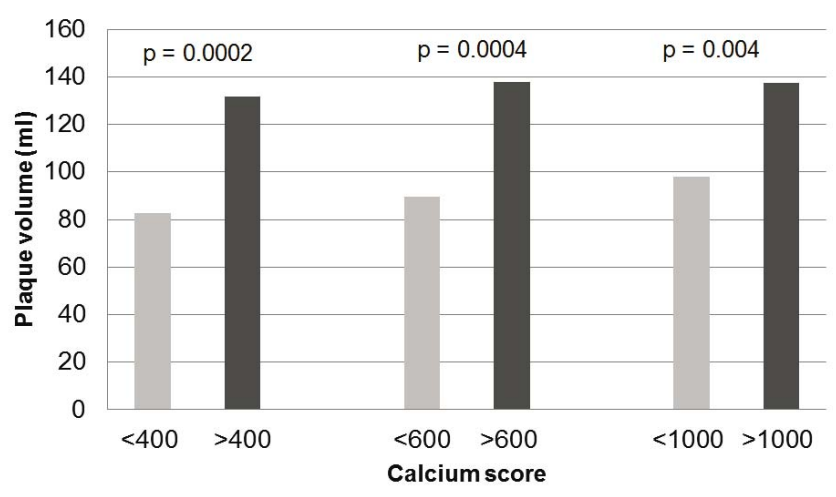

Fig. 1. Plaque volume in different Calcium score cut-off points the lesion $[1,2,3]$. It has been also shown that voluminous plaques together with other features like: positive remodeling index, spotty calcification, presence of low density, are good markers for characterization of a vulnerable plaque. Other authors [4] showed in their comparative study of vulnerable plaque analysis with IVUS and MDSCT a good correlation between vulnerable plaque characteristics and coronary calcium score. The plaque burden was significantly higher in patients with calcium scoring above 400, as well as the external elastic membrane volume, total atheroma volume, percent atheroma volume, absolute dense calcium and necrotic core areas. Another study indicated that a CACS $>400$ was associated with increased risk of significantly obstructive stenosis [5].

Similar to other studies published in the literature [6], our results show that the difference between culprit lesion's
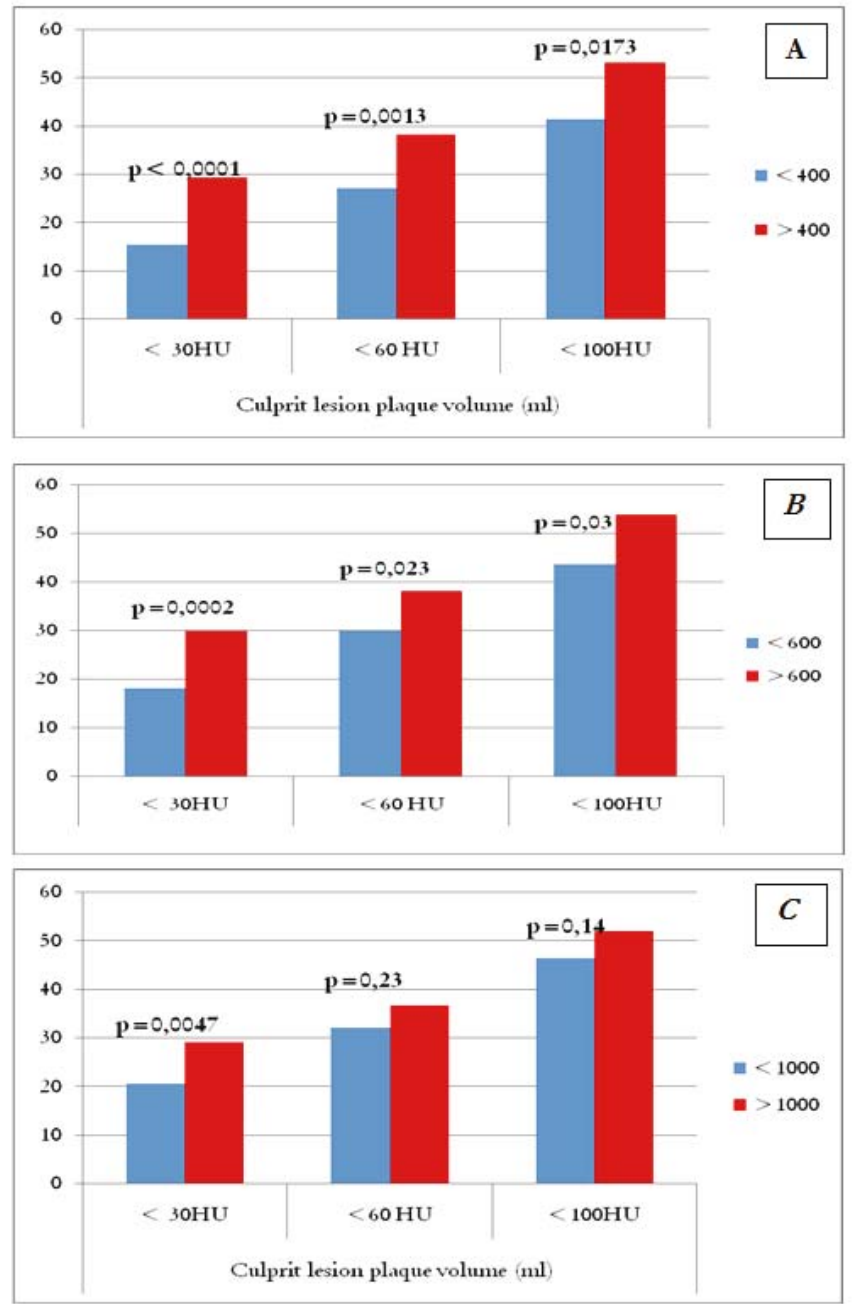

Fig. 2. The culprit lesion different density plaque volume, cut-off point of calcium score. A - 400, B - 600, C - 1000 
plaque volume in patient with calcium score above 1000 and patients with calcium score below 1000 were statistical significant $(p=0.0002)$. In case of a cut-off point of calcium score of 600 or even 400 , the differences between the groups remained statistically significant, even if less expressed.

According to this issue we can conclude that the culprit lesion of a patient with high calcium score is more vulnerable/sever than in patient with low calcium score, thereby this patient more likely develop an acute coronary syndrome, because the coronary artery calcium score is a value of presence of calcium burden within the coronary arteries, which has been showed to have direct correlation with the evolution of the patients towards development of a cardiovascular event. This finding helps the clinician in early detection/prevention in asymptomatic patients with low to intermediate risk score (Recommendations for Calcium Scoring Methods Class IIA, IIB: 2010 ACCF/ AHA Guideline for Assessment of Cardiovascular Risk in Asymptomatic Adults: Executive Summary) [7] and in diagnosis of coronary artery disease in symptomatic patients with low and intermediate risk $[6,8]$. Using this information and assessing our result we had a tendency to manage the intermediate or high risk patients with high calcium score to emergency catheterization laboratory to invasive treatment knowing that this categories of patients is more vulnerable than patients with low calcium score, with less vulnerable lesion.

Some investigators believe that calcium stabilizes the plaque, this information is based on some results that showed that in stable angina the calcium score is higher than in acute coronary syndrome $[6,10]$. It was suggested that CAD patients with high calcium score have more vulnerable plaque components $[8,11]$ (such as higher burden with calcium or presence and extent of the necrotic core within the plaques) than those with low calcium score $[4,10,12]$.Our study results showed that patients with acute coronary syndrome and high calcium score had low attenuation voluminous plaque. Different cut-off value of plaque density: $30 \mathrm{HU}, 60 \mathrm{HU}, 100 \mathrm{HU}$ showed the same change if we assessing patients with calcium score below or above than 400,600, 1000: the culprit lesion plaque mainly is composed by low attenuation $(<30 \mathrm{HU})$ substance, the $\mathrm{p}$ value being statistical significant in all three groups. The increased plaque volume with low density (as the vulnerable plaque characteristics) gives the instability pattern to the culprit lesion.

In the light of these results MDCT presents a particular advantage, allowing to distinguish the high risk ACS patients with a highly unstable plaque from patients in whom the invasive treatment can be delayed.

\section{Conclusions}

Our data shows that patients with high calcium score who develop an acute coronary syndrome present larger atheromatous plaque than those with low calcium scores. Also, the burden with low density atheroma at the site of the culprit lesions is higher in patients with high calcium score especially for low cutoff points of CT density, and the severity of the culprit lesions correlates with the global cardiovascular risk as expressed by a high calcium score.

\section{Acknowledgement}

This paper is partly supported by the Sectorial Operational Programme Human Resources Development (SOP HRD), financed from the European Social Fund and by the Romanian Government under the contract number POSDRU 80641.

\section{Abbreviations}

ACS - Acute Coronary Syndrome

CAD - Coronary Artery Disease

CT - computed tomography

IVUS - Intravascular Ultrasound associated with Virtual Histology

MDCT - 64-slice multidetector computed tomography NSTEMI -non-ST-segment elevation myocardial infarction

UA - unstable angina pectoris

\section{References}

1. BenedekTh, Gyöngyösi M, Benedek I. Multislice Computed Tomographic Coronary Angiography for Quantitative Assessment of Culprit Lesions in Acute Coronary Syndromes. Canadian Journal of Cardiology. 2013;29(3): 364-371.

2. Shapiro E, Bush ED. Visualizing Vulnerability Toward a new cardiac score. J Am CollCardiol. 2013;61(22):2306-2308.

3. Takashi K, Maehara A, Mintz SB, et al. The Dynamic Nature of Coronary Artery Lesion Morphology Assessed by Serial Virtual Histology Intravascular Ultrasound Tissue Characterization. J Am Coll Cardiol. 2010;55(15):1590-1597.

4. Choi YH, Hong JY, Myung H, et al. Relationship between Coronary Artery Calcium Score by Multidetector Computed Tomography and Plaque Components by Virtual Histology Intravascular Ultrasound. J Korean Med Sci. 2011;26(8):1052-1060.

5. Ho JS, Fitzgerald SJ, Stolfus LL, et al. Relation of a coronary artery calcium score higher than 400 to coronary stenoses detected using multidetector computed tomography and to traditional cardiovascular risk factors. Am J Cardiol. 2008;101(10): 444-7.

6. vanWerkhoven JM, Schuijf JD, Gaemperli O, et al. Incremental prognostic value of multi-slice computed tomography coronary angiography over coronary artery calcium scoring in patients with suspected coronary artery disease. Eur Heart J. 2009;30(21):2622-9.

7. Writing Committee Members 2010 ACCF/AHA Guideline for Assessment of Cardiovascular. Circulation. 2010;122:2748-2764.

8. De Feyer JP, Nieman K. CCTA to guide revascularization for high-risk CAD: a cliff hanger. Eur Heart J. 2012;33:3011-3013.

9. Benedek Th, Bucur O, Pascanu I, Benedek I. Analysis of coronary plaque morphology by 64-multislice computed tomography coronary angiography and calcium scoring in patients with type 2 diabetes mellitus. Acta endocrinologica. 2011;7(1):59-68.

10. vanVelzen EJ, Schuijf DJ, de Graaf RF. Diagnostic performance of noninvasive multidetector computed tomography coronary angiography to detect coronary artery disease using different endpoints: detection of significant stenosis vs. detection of atherosclerosis. Eur Heart J. 2011;32 (5):637-645

11. Versteylen OM,Kietselaer LB,Dagnelie CP, et al. Additive value of semiautomated quantification of coronary artery disease using cardiac CTangiography to predict for future acute coronary syndrome. J Am Coll Cardiol. 2013;61(22):2296-305.

12. Yang $X$, Gai LY, Li P, et al. Diagnostic accuracy of dual-source CT angiography and coronary risk stratification. Vasc Health Risk Manag. 2010;6:935-41. 